\title{
Calibration and Performance of the ATLAS Tile Calorimeter During the LHC Run 2
}

\author{
Krystsina Petukhova*, on behalf of the ATLAS collaboration \\ Charles University, Prague, Czech Republic \\ E-mail: krystsina.petukhova@cern.ch
}

The Tile Calorimeter (TileCal) is the central section of the hadronic calorimeter of the ATLAS experiment and provides important information for reconstruction of hadrons, jets, hadronic decays of tau leptons and missing transverse energy. It also assists in muon identification. This sampling calorimeter uses steel plates as absorber and scintillating tiles as active medium. The light produced by the passage of charged particles is transmitted by wavelength shifting fibres to photomultiplier tubes (PMTs). The readout is segmented into about 5000 cells (longitudinally and transversally), each of them being read out by two PMTs in parallel. TileCal exploits several calibration systems: a Cs radioactive source that illuminates the scintillating tiles directly, a laser light system to directly test the PMT response, and a charge injection system (CIS) for the front-end electronics. These systems together with data collected during proton-proton collisions provide extensive monitoring of the instrument and a means for equalizing the calorimeter response at each stage of the signal propagation. The performance of the calorimeter has been established with cosmic ray muons and the large sample of the proton-proton collisions. The response of high-momentum isolated muons is used to study the energy response at the electromagnetic scale, The response of high-momentum isolated muons is used to study the energy response at the electromagnetic scale, isolated hadrons are used as a probe of the hadronic response. The calorimeter time resolution is studied with multijet events. A description of the different TileCal calibration systems and the results on the calorimeter performance during the LHC Run 2 will be presented. The results on the pile-up noise and response uniformity studies with Monte Carlo simulation will be discussed.

XXIX International Symposium on Lepton Photon Interactions at High Energies - LeptonPhoton2019 August 5-10, 2019

Toronto, Canada

${ }^{*}$ Speaker. 


\section{Introduction}

The Tile Calorimeter of the ATLAS experiment [1] at the LHC [2] is composed of 256 wedgeshaped modules, each is a mechanical assembly of the readout cells, front-end electronics (FEE), and elements of the detector control, calibration as well as infrastructure systems. The deposited energy is evaluated based on the measured amplitude $A$ and a set of the calibration coefficients described in Section 2:

$$
E[\mathrm{GeV}]=A[\mathrm{ADC}] \cdot C_{\mathrm{ADC} \rightarrow \mathrm{pC}}^{\mathrm{CIS}} \cdot C_{\mathrm{laser}} \cdot C_{\mathrm{Cs}} \cdot C_{\mathrm{pC} \rightarrow \mathrm{GeV}} \cdot
$$

\section{The TileCal calibration}

The TileCal calibration systems monitor the detector status and provide a means for equalizing the calorimeter response at each stage of the signal propagation [3].

During the Cesium calibration, ${ }^{137} \mathrm{Cs}$ radioactive $\gamma$-sources $(662 \mathrm{keV})$ go through a system of steel tubes traversing all the scintillating tiles. The corresponding calibration constant $C_{\mathrm{Cs}}$ quantifies the produced light yield with respect to the electromagnetic (EM) scale $C_{\mathrm{pC} \rightarrow \mathrm{GeV}}$ determined in beam tests. The Laser system sends a controlled amount of light $(532 \mathrm{~nm})$ to the photocathode of each PMT [4]. An update of the laser calibration constants $C_{\text {laser }}$ addresses the PMT gain drift. The Charge Injection System injects the charge of a known value (between 0 and $800 \mathrm{pC}$ ) into the capacitor and measures the FEE response in ADC-counts $\left(C_{\mathrm{ADC} \rightarrow \mathrm{pC}}^{\mathrm{CIS}}\right)$. The detector-wide RMS variation of $C_{\mathrm{ADC} \rightarrow \mathrm{pC}}^{\mathrm{CIS}}$ over the entire Run 2 is $0.03-0.04 \%$ (Figure 1a). The Minimum Bias system gives information about the instantaneous luminosity and monitors the full optical route.

The response variation is confirmed in the Laser, Cesium, and Minimum Bias calibrations, independently of each other. While the Laser calibration measures only the PMT response degradation due to current, the Minimum Bias calibration additionally identifies the scintillator-light yield loss due to radiation (Figure 1b). This explains the difference between the two responses in Figure 1b. The response down-drifts during physics collisions as a result of the PMT gain loss due to the PMT current and the scintillator aging. During the LHC stops and shutdowns, the response drifts up as the PMT gain recovers.

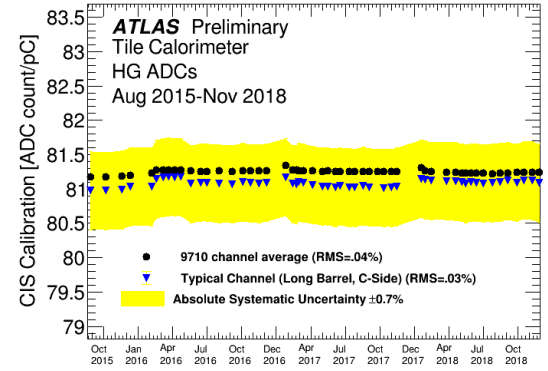

(a)

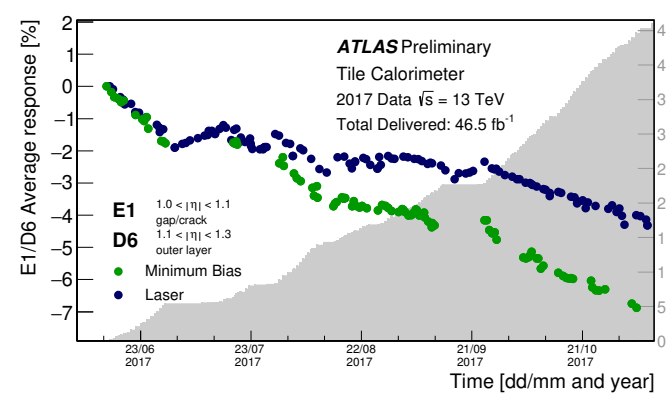

(b)

Figure 1: Evolution of CIS calibration constants in the entire Run 2 (a) [5]. The evolution of response in Minimum Bias and Laser calibrations over 2017 data-taking (b) [5]. The variation of the response of one of the most exposed cells (cell E1) is derived with respect to the response of the least exposed cell (cell D6). 


\section{The TileCal performance}

The response to high-momentum isolated cosmic muons is used to verify the EM scale. Isolated hadrons are used as a probe of the hadronic response [6]. The detector response uniformity (Figure 2a) and linearity are observed.

The electronic noise is measured to be at the level of $20-40 \mathrm{MeV}$. The pile-up noise is measured with zero-bias triggered events and compared to the minimum bias Monte Carlo simulations (Figure 2b). The time resolution studied with multijet events is within $1 \mathrm{~ns}$ for the cell depositing above $30 \mathrm{GeV}$ [7]. The jet energy resolution is within the designed value: $\frac{\sigma}{E}=\frac{50 \%}{\sqrt{E}} \oplus 3 \%$.

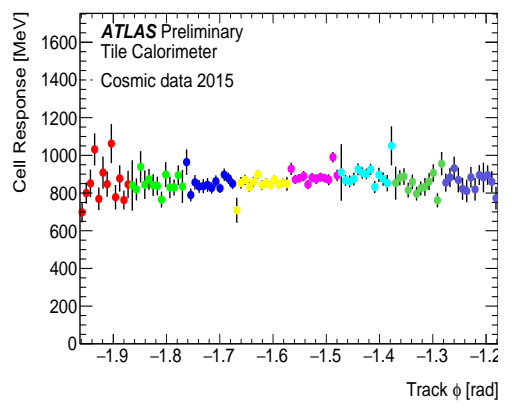

(a)

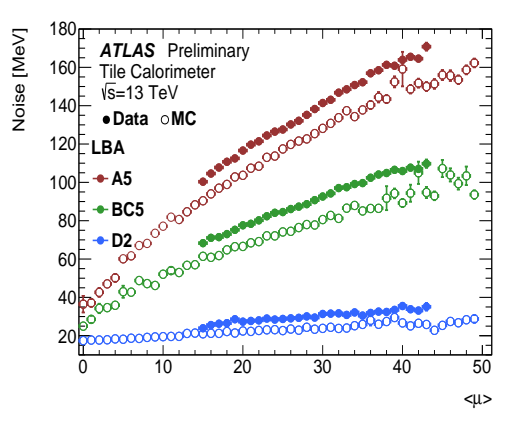

(b)

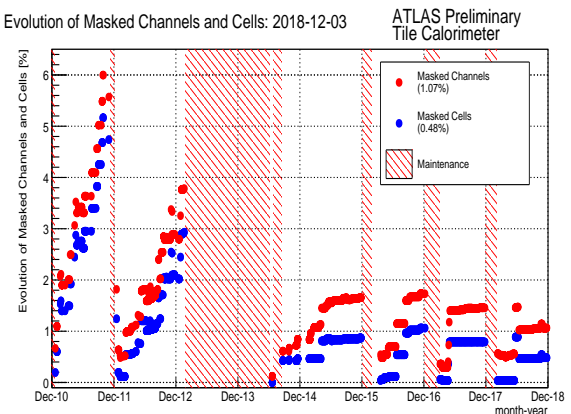

(c)

Figure 2: The profile of cosmic muon energy deposition as a function of the muon track $\phi$ of impact point (a) [8]. The pile-up noise as a function of $\langle\mu\rangle$ [9] shown for one TileCal tower with cells A5, BC5 and D2 in $0.4<\eta<0.5$ (the LBA TileCal partition). The evolution of cell masking (c) [10].

\section{Conclusion}

In LHC Run 2, the TileCal data quality efficiency exceeded 99\%. At the end of Run 2, 0.46\% of cells and $1.05 \%$ of channels were masked (Figure 2c). The TileCal plays a key role in jet and MET reconstruction in the ATLAS experiment and provides the stable (within 1\%) response.

\section{Acknowledgments}

The support of the Visegrad fund and the grant LTT 17018 of the Ministry of Education, Youth and Sport of the Czech Republic is acknowledged.

\section{References}

[1] ATLAS Collaboration, JINST 3 (2008) S08003. 437 p.

[2] L. Evans and P. Bryant, LHC Machine, JINST 3 (2008) S08001.

[3] https://twiki.cern.ch/twiki/bin/view/AtlasPublic/AtlasTechnicalPaperListOfFigures.

[4] ATLAS Tile Collaboration, Journal of Instrumentation 11 (2016) T10005.

[5] https://twiki.cern.ch/twiki/bin/view/AtlasPublic/ApprovedPlotsTileCalibration.

[6] https://twiki.cern.ch/twiki/bin/view/AtlasPublic/ApprovedPlotsTileSingleParticleResponse.

[7] https://twiki.cern.ch/twiki/bin/view/AtlasPublic/TileCaloPublicResultsTiming.

[8] https://twiki.cern.ch/twiki/bin/view/AtlasPublic/ApprovedPlotsTileEnergyCalibration.

[9] https://twiki.cern.ch/twiki/bin/view/AtlasPublic/ApprovedPlotsTileNoise.

[10] https://twiki.cern.ch/twiki/bin/view/AtlasPublic/ApprovedPlotsTileDetectorStatus. 